\title{
TEN YEARS OF COMPACT SYNCHROTRON LIGHT SOURCE AURORA
}

\author{
T. Hori \\ Laboratory for Quantum Equipment Technology, Sumitomo Heavy Industries, Ltd. \\ 1-1 Yato-machi 2-chome, Tanashi, Tokyo 188-8585 Japan
}

\begin{abstract}
From mid 1980's, we have been developing compact SR rings named AURORA. The first one, AURORA-1 (A1), which employed a superconducting magnet to make the ring as compact as possible, has unique features came from its constitution, one circular $360^{\circ}$ bending with no focus magnets. After observed first SR in 1989, we continued to improve the whole system, then transferred to Ritsumeikan Univ. in 1995. It has been in routine operation since then. In parallel with the A1's improvements, we started developing another concept in early 1990's. We adopted normal conducting magnets instead of superconducting's to the second, AURORA-2 (A2), keeping compactness by increasing the bending field, 2.7 Tesla, as high as somewhat comparable to the superconducting's. The most outstanding feature of A2 lies in the bending magnets, whereas the configuration of $\mathrm{A} 2$, racetrack, is very conventional. There are two versions in A2; one pursued compactness, the same purpose as A1, for X-ray lithography, and the other modified for scientific research taking advantage of its capability of accommodating insertion devices (ID's). In 1997, the latter was installed in Hiroshima Univ. as HiSOR in combination with two undulators. On the contrary, the former has been in commissioning at our Tanashi Works.
\end{abstract}

\section{INTRODUCTION}

A compact synchrotron light source has been being developed since mid 1980's from the viewpoint of optimization for industrial use, especially for X-ray lithography. AURORA was originally designed under the trends of those days in applying superconducting technology to such the small SR rings. A1, the world's smallest SR ring, is quite unique by its constitution of a single-body superconducting bending magnet, to which the function of weak-focusing is added to avoid additional focusing magnets. To make the system compact as a whole, a $150-\mathrm{MeV}$ racetrack microtron was newly developed as a reliable and stable injector. The operation of the total system is quite simple, therefore to be suited for industrial application. Extremely small beam size in vertical, originated from very weak $x-y$ coupling, is another attractive feature for scientific experiments.

It proved somehow inadequate to the industrial system, however, because of rather a long maintenance period and recovery time from failure. We thus concluded to adopt room-temperature magnets avoiding superconducting technology in the second version under the restriction of maintaining A1's compactness. It was achieved by an innovative idea of dipole magnets that brought us enough a high magnetic field for normal conducting magnets.
We have some flexibility in the design of new AURORA, depending on how to use straight sections of the racetrack ring. One which pursued compactness by the shortest straight section is called AURORA-2S (A2S), where S means Q- inglet in the straight section. This quadrupole acts on horizontal focusing, whereas edgefocusing on vertical. The other having 3-m free space in the straight section is called AURORA-2D (A2D), where D means Q-Doublet. A2D has 4-sets of the doublet. Two types of ID, undulator and/or wiggler, are available for A2D. A2D in combination with two undulators was constructed in 1997 as HiSOR. Furthermore, the capability to install even a superconducting wiggler in such a compact ring as A2D using the low-energyinjection scheme was also proved by the other A2D.

\section{THE FIRST AURORA (A1) [1-5]}

A1 is the only circular ring in the world, which is the ultimate shape of compactness. The ring's outer diameter is $\sim 3 \mathrm{~m}$, whereas the orbit diameter is $1 \mathrm{~m}$. The ring is unique because of its injection method using half-integer resonance. A1 is one of typical superconducting SR rings of those days, however, which was considered inevitable to produce a compact one. After the end of R\&D, A1 was moved to Ritsumeikan University who established SR Center in 1996.

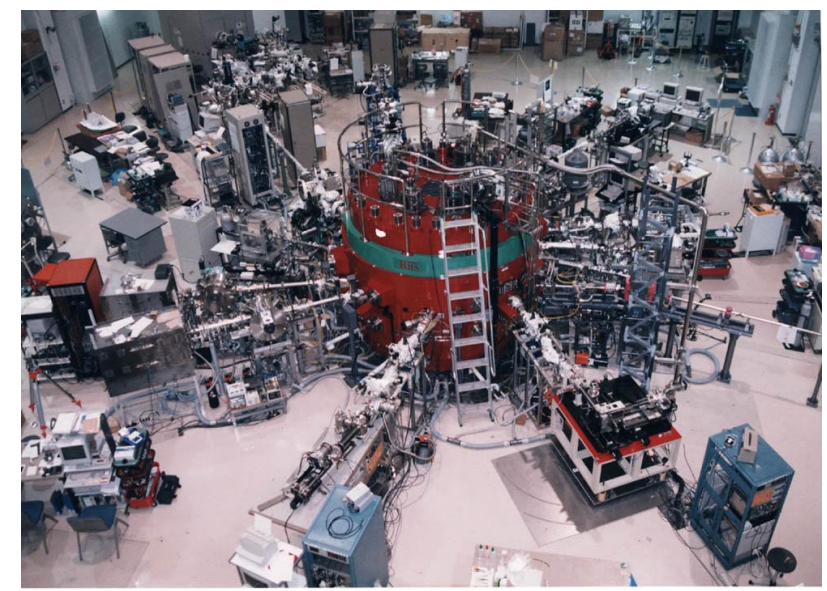

Fig. 1 Whole view of AURORA facility at SR Center in Ritsumeikan University.

Soft X-rays are intensively used for various analyses in material science. Micro-machining is one of unique applications, where LIGA and TIEGA (Teflon Included Etching Galvanicforming) are included. Some of 16 beam lines in total are able to use photons even at $3 \mathrm{~m}$ distance from the source point, which is one of a merit of self-shielding. It means that the users can greatly benefit by a higher photon density than that of other rings. In addition, it was recently measured by 
interferometer that the beam size $\sigma y$ is quite small, $17 \mu \mathrm{m}$, in spite of a larger emittance than $10^{-6} \mathrm{~m} \cdot \mathrm{rad}[6]$. The beam is usually maintained $0.14 \mathrm{~mm}$ of $\sigma y$, however, to avoid too short Touschek lifetime. Thus, the ring is operated twice a day of injection, starting from $300 \mathrm{~mA}$ stored current and terminated at about $150 \mathrm{~mA}$ after 6 hours of accumulation.

\section{THE SECOND AURORA (A2) [7, 8]}

A2 is a compact ring of racetrack type using normalconducting magnets, which takes over many advantages of $\mathrm{A} 1$, the $150-\mathrm{MeV}$ injector microtron [9], cryopanels for high vacuum, self-shielding function, etc.

\subsection{HiSOR - A2D with undulators $[10,11]$}

One A2D with two undulators was built at Hiroshima Synchrotron Radiation Center (HSRC) as HiSOR, which was established in 1997 by Hiroshima University. The most outstanding feature of HiSOR lies in these linear and helical undulators. These devices offer the users higher flux densities than $10^{15}$ photons $/ \mathrm{sec} / \mathrm{mrad}^{2} / 0.1 \%$ b.w. at $300 \mathrm{~mA}$ of the specified current, covering a spectral range from a few to hundreds $\mathrm{eV}$.

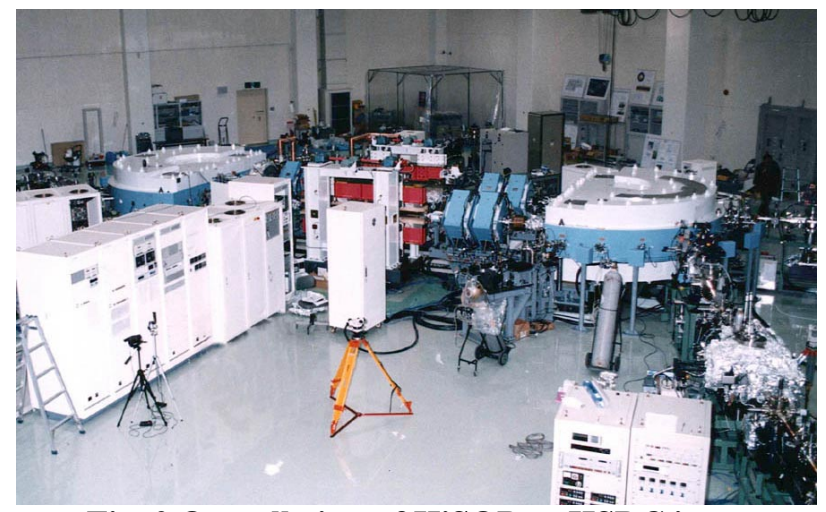

Fig. 2 Overall view of HiSOR at HSRC in Hiroshima University.

In the early stage of A2D's commissioning, we recorded $318 \mathrm{~mA}$ of stored current at $700 \mathrm{MeV}$, starting acceleration from $384 \mathrm{~mA}$ of $150 \mathrm{-MeV}$ injected beam which resulted in $83 \%$ of acceleration efficiency. After the completion of vacuum system upgrading, the beam lifetime was improved to 10 hours at $100 \mathrm{~mA}$, where the vacuum pressure was $1 \times 10^{-9}$ Torr on the average. The operation cycle has reduced to twice a day from three times a day since then. The integrated current has passed beyond $100 \mathrm{~A} \cdot \mathrm{Hr}$. At present, ten of sixteen beam lines, two of them are for undulators, has already been occupied.

\subsection{A2D with 7 Tesla Wiggler [12-15]}

The system check of A2D with a superconducting wiggler was performed in 1997 within a very limited period, $\sim 3$ weeks of net commissioning, prior to the reassembling to $\mathrm{A} 2 \mathrm{~S}$. The test was carried out under the very poor vacuum condition, $\sim 1 \times 10^{-7}$ Torr while beam injection and acceleration, because of no baking and aging process for beam ducts and vacuum chambers. The result was, however, very satisfactory for us.

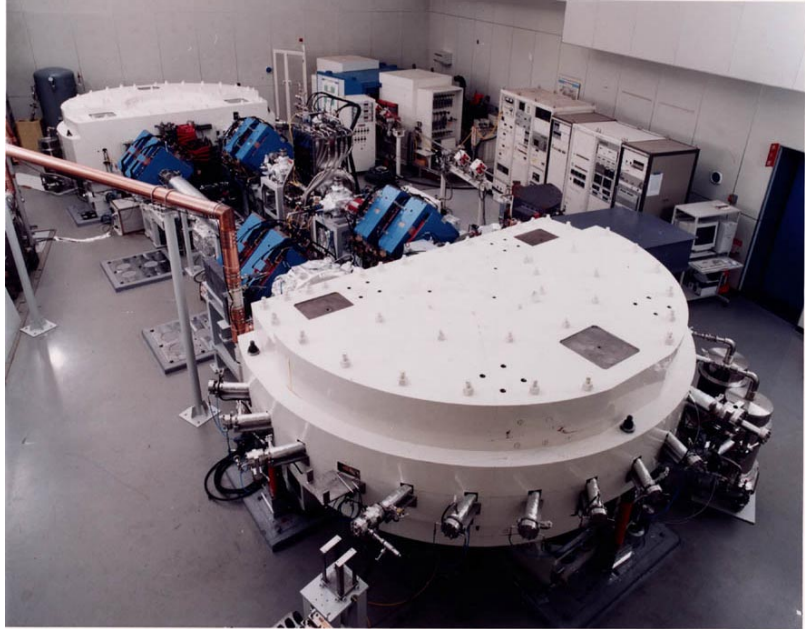

Fig. 3 Photo of A2D with 7 Tesla wiggler taken at our Tanashi Works.

We succeeded in proving the fact that even a compact ring with low-energy-injection scheme like A2D can accommodate a superconducting wiggler as an ID.

The test condition was as follows: The main pole of the wiggler is kept at 1.5 Tesla while injection, and ramped up to 7 Tesla taking 5.5 minutes of acceleration. The accumulated current was limited to $19 \mathrm{~mA}$ because of the poor vacuum in a short test period. We have acquired enough evidence, however, to confirm that the compact ring with 7 Tesla wiggler should work well notwithstanding a large amount of influence on the beam from the wiggler.

\section{$3.3 A 2 S[16,17]$}

A2S is the world's smallest normal conducting SR ring. After the successful test of A2D, it was immediately disassembled and reassembled to A2S in late 1997.

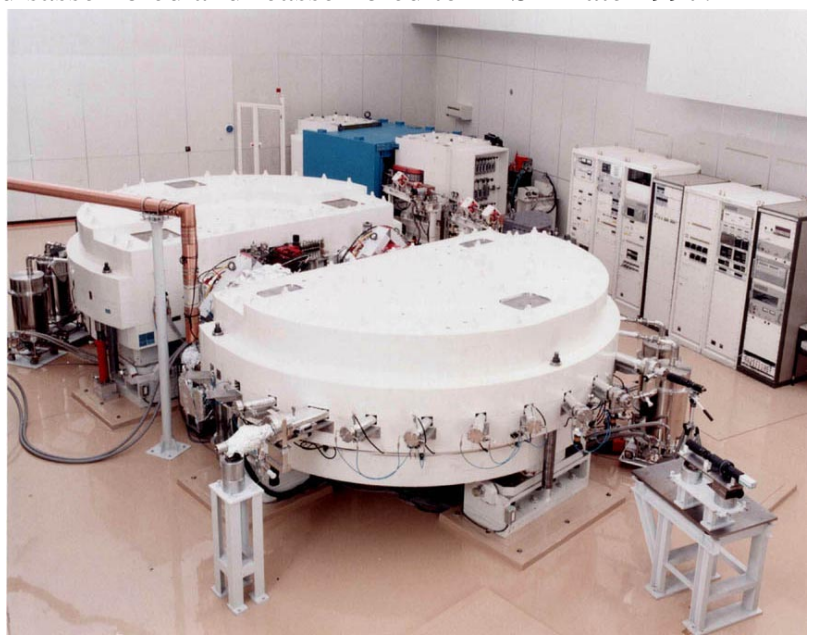

Fig. 4 Photo of A2S before wrapped up in the shielding materials.

Successfully curing the coupled bunch instability using the new RF cavity by which harmful HOM's were greatly suppressed, we went beyond the specification within a month and recorded $719 \mathrm{~mA}$ of stored current so far. Commissioning of A2S has been continued to improve the lifetime, where 3 hours at $500 \mathrm{~mA}$ is observed after $120 \mathrm{~A} \cdot \mathrm{Hr}$ of the integrated current. We 
found no difficulties in the acceleration when ramping more than $800 \mathrm{~mA}$ of injected beam up to $700 \mathrm{MeV}$ in two minutes. The maximum injected beam already exceeded 1A. The reliability check had been done prior to covering the ring with radiation shielding materials as seen in Fig. 5, that is, more than ten days of 24-hours-aday operation terminated successfully.

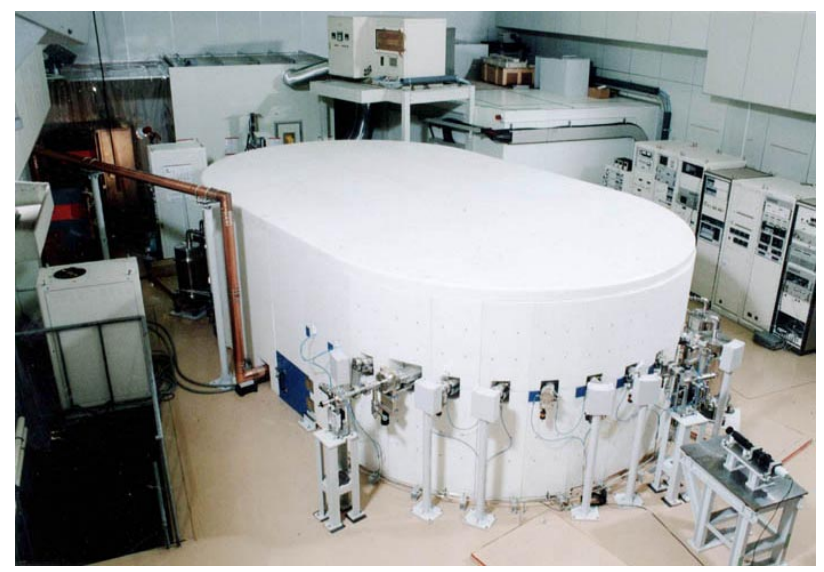

Fig. 5 Photo of A2S after coverd with the polyethylene neutron shielding.

\section{CONCLUSION}

More than a decade, we have been developing compact SR rings for industrial applications. Recently, the applications are extending from X-ray lithography to micromachining such as LIGA and TIEGA. A1 is convenient to obtain high dose rate with ease by placing a target close to the source.

We established a system of easy-handling by eliminating superconducting elements from A2. It was proved to accept either undulators or superconducting wigglers in A2D as ID's, thus even such a small SR ring as HiSOR is quite useful for scientific researches.

Another variation, A2S, has been proved to accumulate much more current than specified. The reliability was checked by a long-term continuous operation. Next step to be proved is its self-shielding capability. The ring is ready for exposure test of X-ray lithography, where a special beam line and a new SR stepper are in the final stage of preparation.

\section{ACKNOWLEDGMENTS}

The author is indebted to Y. Yamamoto of SR Center in Ritsumeikan Univ. for the information of superconducting AURORA, and to $\mathrm{K}$. Goto of HSRC in Hiroshima Univ. for those of HiSOR.

\section{REFERENCES}

[1] N. Takahashi, "Compact Suoerconducting SR Ring for XRay Lithography”, NIM B24/25 (1987) pp.425-428.

[2] T. Takayama, "Resonance Injection Method for Compact Supercond. SR-Ring”, NIM B24/25 (1987) pp.420-424.

[3] H. Yamada, "Commissioning of aurora: The smallest synchrotron light source", J. Vac. Sci. Technol., Vol. B8, 1990, pp.1628-1632.
[4] "SR Center Activity Report", 1996 version /1997 version, Ritsumeikan Univ.

[5] H. Iwasaki, et al., "Compact Superconducting Ring at Ritsumeikan University”, J. Synchrotron Rad. (1998) vol.5, pp.1162-1165.

[6] T. Mitsuhashi, et al., "A Measurement of Beam Size of AURORA by the Use of SR-Interferometer at SR Center of Ritsumeikan University", 11 th Symp. Accel. Sci. Tech., Japan (1997) pp.441-443.

[7] T. Hori, et al., "AURORA-2: Compact Advanced SR Ring as an X-ray Source", 4th Int'l Conf. on SR Sources and 2nd Asian Forum on SR, Korea (1995) pp.148-158.

[8] T. Hori, et al., "Development of New Compact SR Ring AURORA ", APAC'98.

[9] T.Hori, et al., "Improvement of $150 \mathrm{MeV}$ Racetrack Microtron”, PAC'91, pp.2877-2879.

[10] K. Yoshida, et al., "Compact Synchrotron Light Source of the HSRC”, J. Synchrotron, Rad. (1998) pp.1176-1179.

[11] K. Yoshida, et al., "Commissioning of Compact Synchrotron Radiation Source at Hiroshima Univ.", APAC'98.

[12] T. Takayama, et al., "Compact Synchrotron Radiation Source AURORA-2 with 2.7T Normal Conducting Bending Magnets", EPAC'96, pp.709-711.

[13] H. Tsutsui, et al., "Aperture Calculation of AURORA-2D Compact Electron Storage Ring with a Superconducting Wiggler", PAC'97.

[14] T. Hori, "Present Status of AURORA-2D", 11th Symp. Accel. Sci. Tech., Japan (1997) pp.534-536.

[15] T. Hori, et al., "Operation Results of Compact SR Ring AURORA-2D with 7T Wiggler”, EPAC'98, PP.581-583.

[16] H. Miyade, et al., "Initial Commissioning of Dedicated SR Ring AURORA-2S for X-Ray Lithography”, EPAC'98, pp.2413-2415.

[17] H. Miyade, et al., "Beam Test of Compact SR Ring AURORA-2S for X-Ray Lithography”, this conference.

\begin{tabular}{|c|c|c|c|}
\hline & $A 2 S$ & $A 2 D+W$ & Al \\
\hline Energy $(\mathrm{GeV})$ & 0.70 & 0.70 & 0.575 \\
\hline Stored current (A) & $0.5(1.0)$ & 0.3 & 0.3 \\
\hline Circumference (m) & 10.97 & 21.95 & 3.14 \\
\hline RF voltage $(\mathrm{kV})$ & 160 & 220 & 120 \\
\hline Harmonic number & 7 & 14 & 2 \\
\hline RF frequency $(\mathrm{MHz})$ & 191.36 & 191.24 & 190.86 \\
\hline Energy loss (keV/turn) & 24.42 & 29.07 & 19.34 \\
\hline Tune : horizontal & 1.46 & 1.59 & 0.797 \\
\hline Vertical & 0.73 & 2.10 & 0.604 \\
\hline Natural $\varepsilon(\pi \mathrm{mm} \cdot \mathrm{rad})$ & 0.528 & 0.935 & 1.68 \\
\hline Radiation damping : & & & \\
\hline horizontal (msec) & 2.13 & 5.60 & 1.3 \\
\hline vertical (msec) & 2.10 & 3.53 & 0.43 \\
\hline longitudinal (msec) & 1.04 & 1.49 & 0.16 \\
\hline Bunch length (mm) & 26.5 & 36.2 & 52.0 \\
\hline Touschek life (hour) & 20. & 9.9 & $*(0.5)$ \\
\hline Bending field : B (T) & 2.7 & 2.7 & 3.8 \\
\hline n-value & 0.0 & 0.0 & 0.365 \\
\hline $\mathrm{QF}(\mathrm{T} / \mathrm{m})$ & 12.5 & 10.9 & - \\
\hline $\mathrm{QD}(\mathrm{T} / \mathrm{m})$ & - & -12.3 & - \\
\hline
\end{tabular}

Table 1 Parameters of A2S, A2D+wiggler, and A1. *under the extremely small $\varepsilon x-\varepsilon y$ coupling $\left(\sim 2 \times 10^{-4}\right)$ 